\title{
EFEITO DE BORDA EM UM TRECHO DA FLORESTA OMBRÓFILA MISTA, EM FAZENDA RIO GRANDE, PR
}

\author{
Effect of Edge in a Stretch of Araucaria Forest, in \\ Fazenda Rio Grande, PR
}

\author{
Eduardo Malchow ${ }^{1}$ \\ Alexandre Bernardi Koehler ${ }^{2}$ \\ Sylvio Péllico Netto ${ }^{3}$
}

\section{Resumo}

Várias mudanças ecológicas estão associadas às abruptas bordas artificiais dos fragmentos de floresta. O presente trabalho teve por objetivo descrever as principais mudanças florísticas e estruturais em bordas de fragmentos florestais, na Fazenda Experimental Gralha Azul, município de Fazenda Rio Grande, Estado do Paraná. Para tal foram instaladas 100 parcelas amostrais, subdivididas em sete blocos no sentido da borda para o interior, cada uma com cinco metros de comprimento por 10 metros de largura. Os parâmetros analisados foram a composição florística e a estrutura em cada bloco e no total. Até os 20 metros em relação à borda constatou-se a presença de espécies pioneiras e secundárias iniciais, como Vernonia discolor, Araucaria angustifolia, Capsicodendron dinisii, Schinus terenbinthifolius, Lithraea brasiliensis, Piptocarpha angustifolia, Ocotea puberula, Clethra scabra; verificou-se também um maior índice de mortalidade. Na região de transição entre o ambiente heliófilo e o umbrófilo, verificou-se a ocupação por espécies como Matayba elaeagnoides, Ilexparaguariensis e Psychotria suterella, todas espécies de sub-bosque. Dos 21 aos 35 metros a taxa de ocupação de espécies pioneiras foi reduzida drasticamente, sendo substituídas por espécies umbrófilas como Myrcia hatschbachii, Myrcia rostrata, Myrcia fallax, Ilexparaguariensis, Eugenia cereja e Eugenia neoverrucosa. Como espécie indiferente à intensidade luminosa e sombreamento constatouse a presença de Capsicodendron dinisii em todos os blocos. Assim, pode-se concluir que o efeito de borda causa um efeito dinâmico na floresta, alterando a composição florística da bordadura, e também um aumento na mortalidade de árvores jovens e adultas.

Palavras-chave: Fragmentos florestais; Estrutura florística; Efeito de borda.

1 Engenheiro Agrônomo da Pontifícia Universidade Católica do Paraná. malchow.@gmail.com

2 Engenheiro Florestal, M.Sc., Prof. Assistente, Pontifícia Universidade Católica do Paraná; alexandre.koehler@pucpr.br

3 Engenheiro Florestal, Dr. Prof. Titular, Pontifícia Universidade Católica do Paraná; E-mail: pellico.sylvio@pucpr.br 


\section{Abstract}

Some ecological changes are associated to the abrupt artificial edges of the forest fragments. The present work had as objective to describe the main floristic and structural changes in the forest fragments edges, in the Experimental Gralha Azul Farm, municipality of Fazenda Rio Grande, Parana State. For that, 100 plots had been installed, subdivided in seven blocks in the direction from the edge to the inside of the forest, each one with five meters of length for 10 meters of width. The analyzed parameters had been the floristic and structure composition in each block and in the total. Until the 20 meters in relation to the edge it was evidenced presence of pioneering species and secondary ones, as Vernonia discolor, Araucaria angustifolia, Capsicodendron dinisii, Schinus terenbinthifolius, Lithraea brasiliensis, Piptocarpha angustifolia, Ocotea puberula, Clethra scabra; a higher mortality rate was also verified. In the transition region between the heliofilus and ombrofilus environment, it was verified the occupation by species as Matayba elaeagnoides, Ilex paraguariensis and Psychotria suterella, all under store species. From 21 to 35 meter the pioneer species occupation rate was drastically reduced, being substituted by ombrofilus species as Myrcia hatschbachii, Myrcia rostrata, Myrcia fallax, Ilexparaguariensis, Eugenia cereja and Eugenia neoverrucosa. As not influenced species to the light intensity and under shade it was evidenced the presence of Capsicodendron dinisii in all blocks. Thus, it can be concluded that the edge effect provokes a dynamic effect in the forest, modifying its floristic composition, and also an increase rate in the mortality of mature and young trees.

Keyword: Forest fragments; Floristic structure; Edge effect.

\section{Introdução}

O processo de fragmentação de ecossistemas inicia-se com a formação de clareiras ou agressões na vegetação original. Inicialmente a composição florística e estrutural do ecossistema permanecem relativamente inalteradas dentro da porção remanescente, mas à medida que os vazios aumentam e se rompe a conectividade da vegetação original, todo o sistema biológico é alterado (MEFFE, 1994).

Após o desmatamento, os fragmentos ficam isolados e sua vizinhança passa a não ser mais a própria floresta contínua, mas áreas abertas, como lavouras, pastagens e estradas. Há, então, uma influência mútua entre os dois tipos de ambientes, e as espécies da floresta respondem de diversas maneiras a esse fenômeno, denominado efeito de borda (TABARELLI et al., 2004).

Os fragmentos diferem do hábitat original de duas maneiras importantes. Primeiramente os fragmentos têm uma quantidade maior de borda por área de hábitat, e segundo, o centro de cada fragmento de hábitat está mais próximo dessa borda (PRIMACK; RODRIGUES, 2001).

Florestas fragmentadas recentemente são fortemente influenciadas pelos efeitos de borda, que são compostos por várias mudanças ecológicas associadas às abruptas bordas artificiais dos fragmentos de floresta. A radiação solar e os ven- tos quentes e secos penetram na floresta, vindos da pastagem que circundam os fragmentos, ocasionando a alteração do microclima da floresta (LAURANCE ${ }^{4}$, citado por PRIMACK; RODRIGUES, 2001).

Devido ao aumento da turbulência e do estresse hídrico, as taxas de mortalidade de árvores aumentam de forma drástica próximo às bordas, ocasionando o aumento intenso de clareiras que alteram a estrutura e a composição da floresta (LAURENCE et al., 1998).

A fragmentação e o efeito de borda induzem um aumento na mortalidade de árvores jovens pela competição por cipós, espécies parasitas e espécies adaptadas a solos pobres, ao mesmo tempo em que a própria derrubada causa um prejuízo físico. Até mesmo árvores adultas ficam mais vulneráveis à borda e, freqüentemente, caem com a elevação de suas raízes para a superfície e com a ação do vento. Além disso, a eliminação de vertebrados dispersores de sementes compromete a reprodução de muitas espécies (TABARELLI et al., 2004).

Um estudo de bordas realizado no norte do Paraná por Rodrigues (1998) mostrou que a largura do efeito de borda é de 35 metros, pois a similaridade de espécies, a densidade de plantas e o déficit de pressão de vapor demandaram essa mesma largura para uma apropriada avaliação.

Os efeitos decorrentes da borda podem ser classificados em efeitos físicos ou abióticos e

\footnotetext{
${ }^{4}$ Laurance, W. F. Projeto de Dinâmica Biológica de Fragmentos Florestais (não publicado).
} 
os biológicos diretos ou indiretos. Os efeitos abióticos mais considerados são a radiação solar, a umidade e o vento. Os efeitos biológicos diretos são avaliados considerando as alterações causadas pelos efeitos abióticos na comunidade, como o aumento da densidade de plantas em função do incremento na radiação solar. Por sua vez, os efeitos biológicos indiretos envolvem mudanças na interação entre as espécies, como predação, parasitismo, herbivoria, competição e dispersão de sementes e polinização (RODRIGUES, 1998).

Esta pesquisa teve como objetivo avaliar o efeito de bordas na composição florística e estrutura dos remanescentes florestais da Fazenda Experimental Gralha Azul.

\section{Material e métodos}

O presente trabalho de pesquisa foi desenvolvido experimentalmente na Fazenda Experimental Gralha Azul, de propriedade da Pontifícia Universidade Católica do Paraná, localizada no Primeiro Planalto Paranaense, Região Metropolitana de Curitiba, município de Fazenda Rio Grande. Situa-se entre as seguintes coordenadas geográfi-

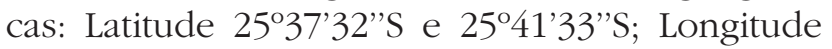

49 15'29'W e 49 17'27" W. Situada em altitudes entre 870 e 920 m, pertence à Bacia Hidrográfica do Alto Iguaçu e possui uma área de 876,4 ha. A classificação climática, segundo o IAPAR (2005), é $\mathrm{Cfb}$, ou seja, clima temperado, com verão ameno, chuvas uniformemente distribuídas, sem estação seca e a temperatura média do mês mais quente não chega a $22^{\circ} \mathrm{C}$, com precipitação de $1.100 \mathrm{a}$ $2.000 \mathrm{~mm}$. Geadas severas são freqüentes, em um período médio de ocorrência de dez a vinte e cinco dias anualmente.

Esta pesquisa sobre efeito de borda foi realizada como um subprojeto no âmbito do projeto PELD/CNPq, Site 9, iniciado na Fazenda Experimental Gralha Azul, desde o ano de 2002.

Para a coleta de dados foram demarcadas 100 parcelas de estudo, dimensionadas no tamanho de 10 metros de largura por 35 metros de comprimento, sendo subdivididas fisicamente com tubos de PVC de $40 \mathrm{~mm}$ de diâmetro, localizados na linha central da parcela, de forma que os 35 metros de comprimento deram origem a sete blocos de dez metros de largura por cinco metros de comprimento, como pode ser observado na Figura 1. A distância mínima entre as parcelas foi de 200 metros, na linha de bordadura dos fragmentos.

FIGURA 1 - Parcela para estudo do efeito de borda.

Figure 1 - Plot used for the edge effect study.

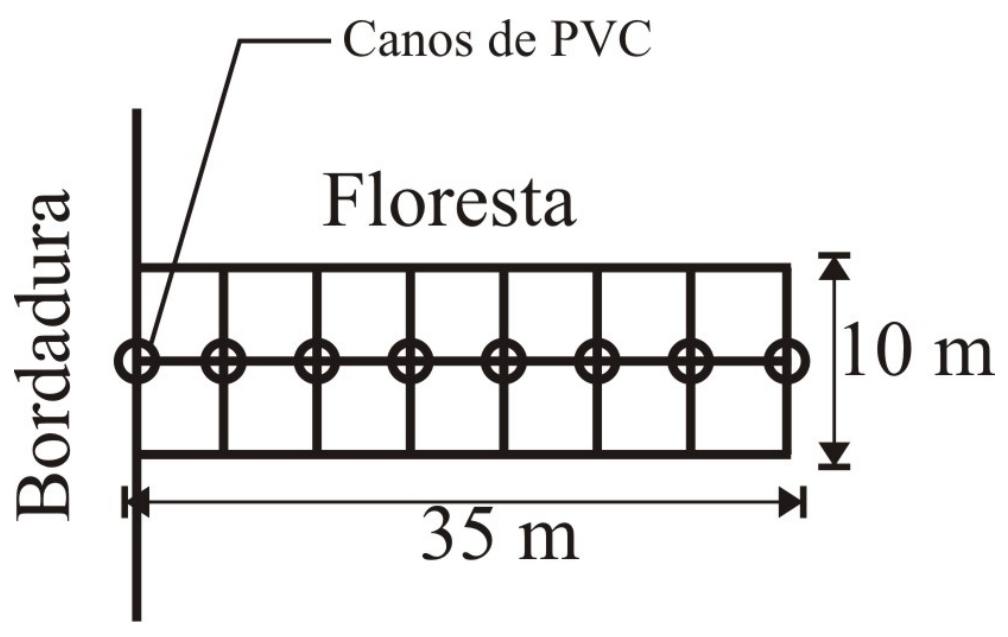


A identificação das espécies de árvores presentes na amostra foi realizada por coleta de ramos, flores e frutos. A composição florística foi avaliada de acordo com o distanciamento da borda da floresta, ao longo dos sete blocos de cinco metros.

Em cada parcela, todas as árvores com DAP (diâmetro à altura do peito, 1,30 m) igual ou maior do que $10 \mathrm{~cm}$ foram marcadas e nelas foram medidas, além do diâmetro, a altura comercial, a altura total e a posição dentro do estrato florestal. Também foi realizado o georreferenciamento das parcelas, para se obter uma dinâmica da distribuição espacial delas dentro da área em estudo.

Os parâmetros para avaliação da estrutura da floresta foram basicamente densidade, dominância, freqüência e valor de importância, bem como foi feita a análise da composição florística e sua variabilidade entre e dentro das unidades amostrais, de acordo com seu afastamento em relação à bordadura da floresta. A densidade foi calculada pela área basal ocupada por cada espécie, sendo expressa em m_/ha. A freqüência refere-se ao número de parcelas em que cada espécie ocorre. A dominância foi calculada pelo número de exemplares de cada espécie em relação ao total de árvores em cada bloco. O valor de importância resultou da somatória da densidade, freqüência e dominância. Para o cálculo da similaridade florística entre os blocos, utilizou-se o coeficiente de Sorensen, conforme Valentin (2000).

Para melhor visualização dos resultados foram gerados gráficos baseados no valor de im- portância de cada espécie, em cada bloco. Além disso, os resultados foram comparados com outros estudos realizados na mesma formação florestal, porém em situações livres do efeito de borda.

Para se identificar possíveis grupos nos blocos estudados foi aplicada a análise de grupamentos (VALENTIN, 2000). De acordo com o método de ligação e a distância utilizada, o critério de formação dos grupos pôde ser bem rigoroso. A adoção do método de Ward para a análise de agrupamentos mostrou-se o mais adequado entre todos os modelos comparativos testados. Este é um método de agrupamento que forma grupos de maneira a atingir sempre o menor erro interno entre os vetores que compõem cada grupo e o vetor médio do grupo. Isso equivale a buscar o mínimo desvio-padrão entre os dados de cada grupo (DUTRA et al., 2004).

\section{Resultados e discussão}

Na Tabela 1 pode-se observar que, com o aumento da distância da bordadura, há uma redução no número de árvores mortas, o que se deve à atenuação da intensidade do efeito de borda, como a incidência de ventos e afloramento das raízes, como verificado por Tabarelli et al. (2004). Há também um sensível aumento no diâmetro médio e altura total das árvores em direção ao interior da floresta, devido ao maior porte das árvores, e redução da intensidade de ocorrência de espécies pioneiras.

\section{TABELA 1 - Parâmetros estruturais em relação ao distanciamento da bordadura.} Table 1 - Structural parameters in relation to the edge remoteness.

\begin{tabular}{lrrrrrrr}
\hline \multirow{2}{*}{ Variável } & \multicolumn{7}{c}{ Distância em relação à bordadura (m) } \\
\cline { 2 - 7 } & $\mathbf{5 , 0 0}$ & $\mathbf{1 0 , 0 0}$ & $\mathbf{1 5 , 0 0}$ & $\mathbf{2 0 , 0 0}$ & $\mathbf{2 5 , 0 0}$ & $\mathbf{3 0 , 0 0}$ & $\mathbf{3 5 , 0 0}$ \\
\hline Número de spp. & 66,00 & 62,00 & 64,00 & 63,00 & 56,00 & 53,00 & 59,00 \\
DAP médio (cm) & 19,69 & 18,23 & 20,32 & 20,19 & 21,28 & 20,82 & 22,73 \\
Altura de corte média (m) & 3,77 & 4,40 & 4,42 & 4,72 & 3,99 & 4,31 & 4,44 \\
Altura total média (m) & 9,89 & 10,57 & 11,20 & 11,25 & 11,53 & 11,78 & 13,03 \\
g (área basal) (m_/ha) & 32,03 & 29,08 & 35,43 & 35,70 & 41,52 & 31,20 & 32,32 \\
Arv/ha & 836,00 & 920,00 & 838,00 & 832,00 & 660,00 & 708,00 & 614,00 \\
Mortas/ha & 66,00 & 54,00 & 72,00 & 64,00 & 66,00 & 42,00 & 26,00 \\
\hline
\end{tabular}


Na Figura 2 foi estabelecido o ordenamento de acordo com o Valor de Importância (VI) de cada espécie, em cada quadrante, sendo que Vernonia discolor, Araucaria angustifolia, Capsicodendron dinisii, Schinus terenbinthifolius e Myrcia hatschbachii ocorrem com VI acima da média nos 35 metros amostrados; Capsicodendron dinisii se mostra uma espécie indiferente. Ressalta-se que a ocorrência de Vernonia discolor após os $20 \mathrm{~m}$ deve-se a fatores ocasionais que favoreceram seu desenvolvimento, como clareiras que permitiram maior entrada de luz. De acordo com Koehler et al. (1998), na análise da estrutura de uma Floresta Ombrófila Mista, realizada na Fazenda Experimental Gralha Azul, a Araucaria angustifolia não se situou entre as espécies mais abundantes, fato que pode ser explicado pela necessidade de uma alta incidência luminosa para seu desenvolvimento. Como observado neste trabalho, a bordadura da floresta é uma região com as condições propícias para sua expansão, podendo ser uma fonte para reestruturação da floresta, já que no interior dos fragmentos há uma baixa ocorrência desta espécie, devido à retirada extrativista em períodos anteriores.

FIGURA 2 - Ordenamento das espécies com presença em todos os blocos, de acordo com o Valor de Importância. As barras brancas representam valores de importância abaixo da média, as barras cinzas indicam o VI médio, e as barras pretas VI acima da média.

Figure 2 - Species ordering in all the blocks, in accordance with the Importance Value (VI). The white bars represent VI below of the average, the bars leached ashes indicate the average VI, and black bars indicate VI above of the average.

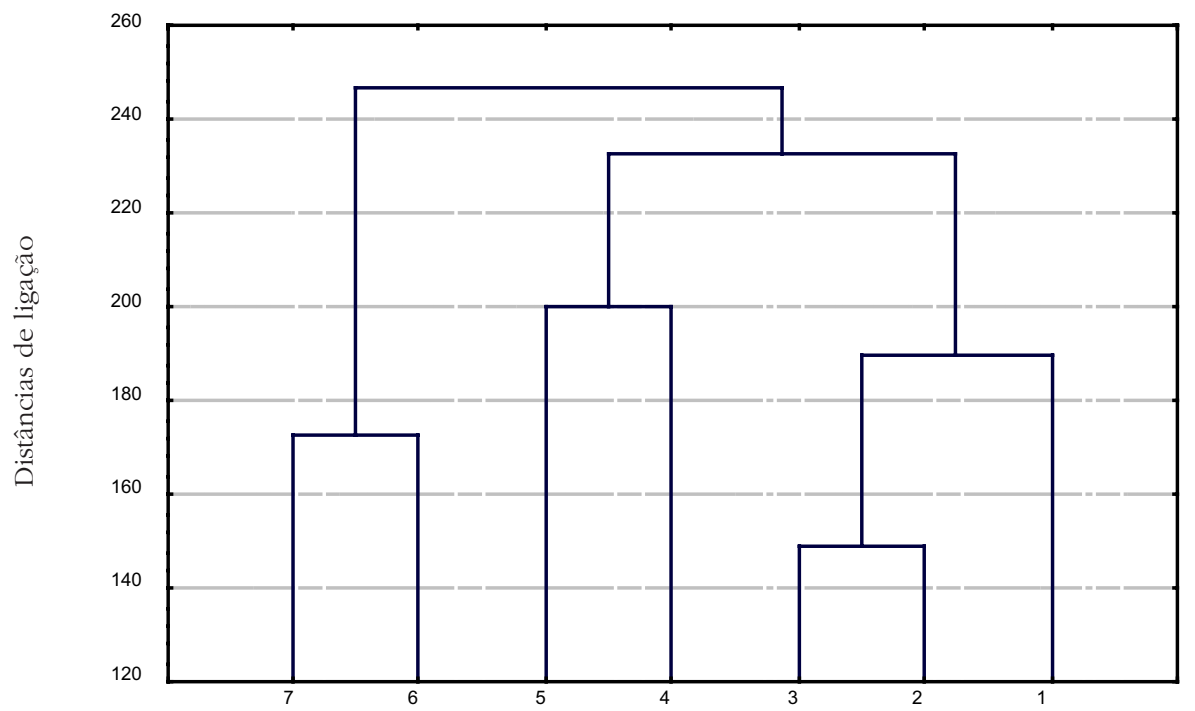

As espécies com maiores valores de importância até os $15 \mathrm{~m}$ da borda da floresta foram Zanthoxylum rhoifolium, Aegiphila sellowiana, Ocotea puberula, Psychotria suterella, Clethra scabra, Cedrela fissilis, Piptocarpha angustifolia e Lithraea brasiliensis, que segundo Lorenzi (2002) são heliófilas e pioneiras, favorecidas pela alta incidência luminosa causada pelo efeito de borda.

As espécies que ocorreram com maior representatividade após os $15 \mathrm{~m}$ foram Casearia decandra, Casearia syluestris, Mlexparaguariensis, Matayba elaeagnoides e Nectandra megapotamica, que ocorrem preferencialmente nos sub-bosques dos pinhais e em formações secundárias, como citado por Lorenzi (2002).

Eugenia cereja, Eugenia neoverrucosa e
Myrcia rostrata são espécies caracteristicamente umbrófilas, estando presentes com maior expressividade entre os 20 e $35 \mathrm{~m}$ da bordadura.

$\mathrm{Na}$ Figura 3, Ocotea odorifera apresenta distribuição crescente para o interior do fragmento, devido as melhores condições de sombreamento para o seu desenvolvimento. Myrsine ferruginea apresenta maior VI dos 5 aos $20 \mathrm{~m}$, pois, segundo Lorenzi (2002), é uma espécie heliófila, ocorrendo em capoeiras, áreas abertas e submata de pinhais. Sapium glandulatum ocorreu com maior intensidade até os $15 \mathrm{~m}$, pois, como citado por Lorenzi (2002), é uma espécie heliófila, encontrada com freqüência em sub-bosques de pinhais parcialmente devastados. Sloanea monosperma tem distribuição regular nos 35 
m de bordadura, não sendo constatada apenas entre os 20 a $25 \mathrm{~m}$, devido a fatores ocasionais que impediram seu desenvolvimento. Gochnatia polymorpha é caracteristicamente pioneira e heliófila e, em conseqüência disso, ocorre com grande expressividade até os $10 \mathrm{~m}$.

FIGURA 3 - Ordenamento das espécies que estiveram ausentes em pelo menos um bloco, de acordo com o Valor de Importância.

Figure 3-Species ordering that had been absent in at least one block, in accordance with the Importance Value.

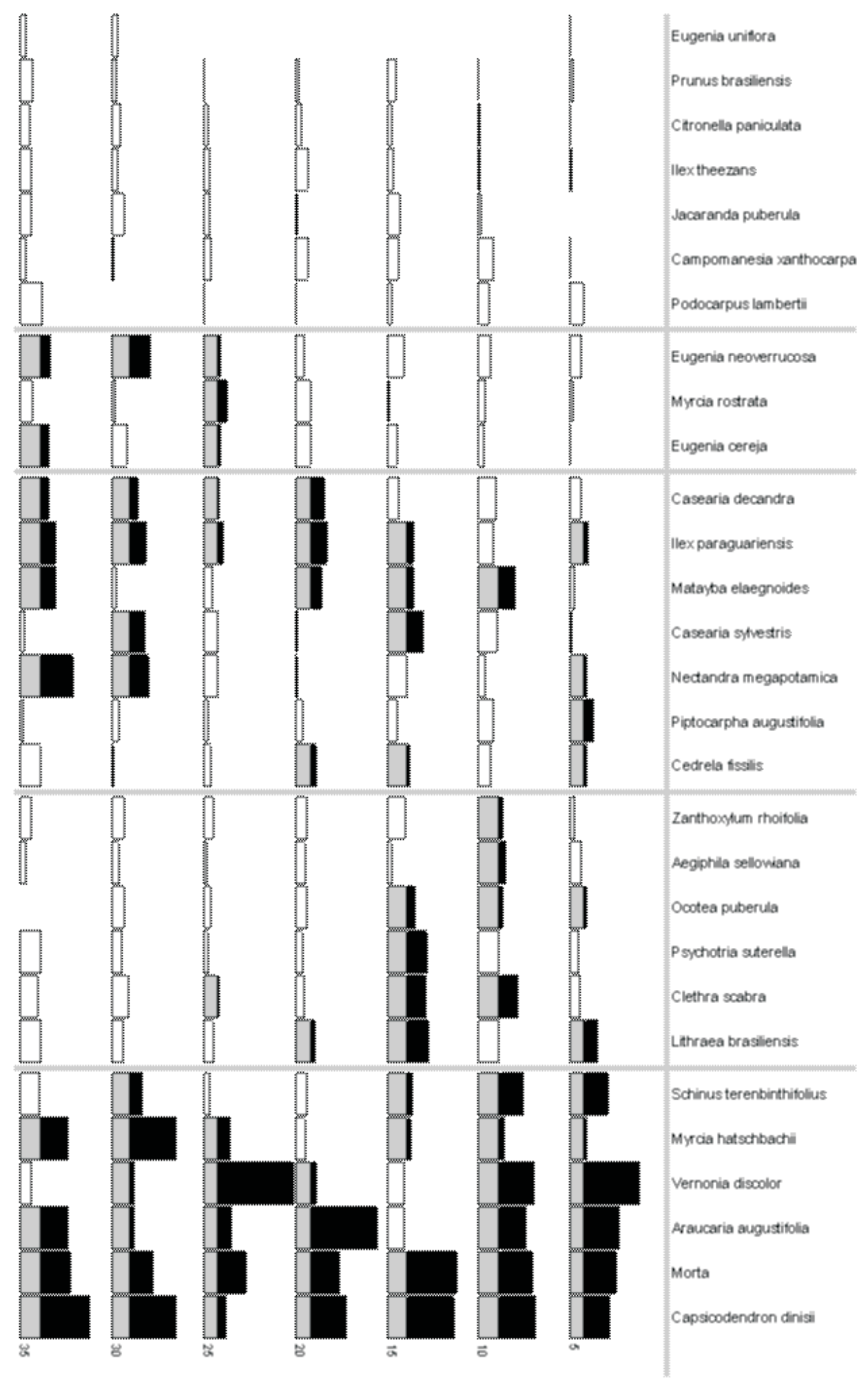


Na Tabela 2 verifica-se que as espécies mais importantes do trecho de floresta amostrado são heliófilas, ou pioneiras, como Vernonia discolor, Schinus terebinthifolius, e a própria Araucaria angustifolia. Isso demonstra que a borda da floresta apresenta uma estrutura diferenciada quando comparada ao interior da floresta, onde predominam espécies secundárias e clímax (KOEHLER et al., 1998). Segundo os autores, na floresta livre do efeito de borda predominam Nectandra megapotamica, Ocotea odorifera e Ilexparaguariensis.

TABELA 2 - Classificação das 20 espécies com maiores Valores de Importância média em todos os blocos (0 a 35m).

Table 2 - The 20 species classified with higher average of Importance Values in all the blocks $(0-35 \mathrm{~m})$

\begin{tabular}{ll|ll}
\hline Espécies & VI (médio) & Espécies & VI (médio) \\
\hline \multirow{2}{*}{ Capsicodendron dinisii } & 19,10 & Casearia decandra & 8,11 \\
Vernonia discolor & 16,93 & Matayba elaeagnoides & 7,79 \\
Araucaria angustifolia & 15,35 & Casearia sylvestris & 6,84 \\
Myrcia hatschbachii & 12,17 & Psychotria suterella & 6,57 \\
Schinus terenbinthifolius & 10,13 & Ocotea puberula & 6,39 \\
Ilexparaguariensis & 10,13 & Cedrela fissilis & 6,35 \\
Lithraea brasiliensis & 8,83 & Eugenia cereja & 5,84 \\
Nectandra megapotamica & 8,82 & Zanthoxylum rhoifolium & 5,82 \\
Clethra scabra & 8,42 & Piptocarpha angustifolia & 5,09 \\
Eugenia neoverrucosa & 8,18 & Myrcia rostrata & 4,70 \\
\hline
\end{tabular}

Floristicamente não ocorrem diferenciações pronunciadas entre os blocos, conforme está apresentada na Tabela 3. Ainda assim, se observada a similaridade entre o bloco $5 \mathrm{~m}$ e os demais, percebe-se tendência regressiva, na medida em que se afasta da borda. A Tabela 4 quantifica as distâncias entre os grupos formados, de acordo com o método de Ward (DUTRA et al., 2004).

TABELA 3 - Similaridades florísticas de Sorensen entre os blocos.

Table 3 -Floristic similarities of Sorensen between the blocks.

\begin{tabular}{lllllll}
\hline Amostras & $\mathbf{5}$ & $\mathbf{1 0}$ & $\mathbf{1 5}$ & $\mathbf{2 0}$ & $\mathbf{2 5}$ & $\mathbf{3 0}$ \\
\hline 10 & 0,75 & & & & & \\
15 & 0,67 & 0,75 & & & & \\
20 & 0,72 & 0,79 & 0,76 & & & \\
25 & 0,61 & 0,69 & 0,62 & 0,68 & & \\
30 & 0,65 & 0,77 & 0,65 & 0,68 & 0,65 & 0,65 \\
35 & 0,64 & 0,73 & 0,73 & 0,67 & 0,55 & \\
\hline
\end{tabular}


TABELA 4 - Distâncias de Ward para os grupos formados, de acordo com o valor de importância de todas as espécies.

Table 4 -Ward Distances for the formed groups, in accordance with the Importance Value for all the species.

\begin{tabular}{lllllll}
\hline Amostras & 5 & 10 & 15 & 20 & 25 & 30 \\
\hline 10 & 153,0 & - & - & - & - & - \\
15 & 206,0 & 149,0 & - & - & - & - \\
20 & 208,0 & 172,0 & 202,0 & - & - & - \\
25 & 217,0 & 190,0 & 234,0 & 201,0 & - & - \\
30 & 235,0 & 195,0 & 187,0 & 222,0 & 209,0 & - \\
35 & 228,0 & 192,0 & 188,0 & 186,0 & 229,0 & 173,0 \\
\hline
\end{tabular}

Levando-se em consideração o valor de importância, observa-se na Figura 4 a formação de um grupo entre os 5 e $15 \mathrm{~m}$, outro grupo dos 20 aos $25 \mathrm{~m}$ e um terceiro grupo dos 30 aos $35 \mathrm{~m}$, sendo que esses agrupamentos refletem o sentido de mudança estrutural rumo ao interior da floresta; bem como uma mudança florística conforme pode ser analisado nas Figuras 2 e 3 .

\section{Conclusões}

1. A borda da floresta apresenta estrutura diferenciada, quando comparada com o interior da floresta, onde predominam as espécies secundárias e clímax.

2. Considerando-se os Valores de Importância (VI) das espécies obtidos a partir da análise fito sociológica, observa-se a formação de um grupo entre 5 e $15 \mathrm{~m}$, outro grupo dos 20 aos $25 \mathrm{~m}$ e um terceiro dos 30 aos $35 \mathrm{~m}$, configurando-se uma mudança florística devido às suas características, como espécies pioneiras ou heliófilas.

3. As variáveis diâmetro médio e altura total das árvores amostradas em direção ao interior da floresta experimentaram sensíveis aumentos devido ao maior porte das árvores e redução de ocorrência de espécies pioneiras.

4. Pesquisas mais aprofundadas que incluam avaliações de variáveis ambientais poderão proporcionar um melhor entendimento do efeito de borda, bem como quantificar sua extensão rumo ao interior da floresta.

\section{Agradecimentos}

À administração da Fazenda Experimental Gralha Azul, bem como ao Prof. Sylvio Péllico Netto pelo apoio incondicional à realização do presente estudo. Agradecemos também ao Sr. Luiz Carlos de Oliveira Santos, funcionário da PUCPR, pelo auxílio nos trabalhos de identificação das árvores em campo. 
FIGURA 4 - Análise de agrupamentos para os blocos, de acordo com o Valor de Importância. Figure 4 - Grouping analysis in the blocks, in accordance with the Importance Value.

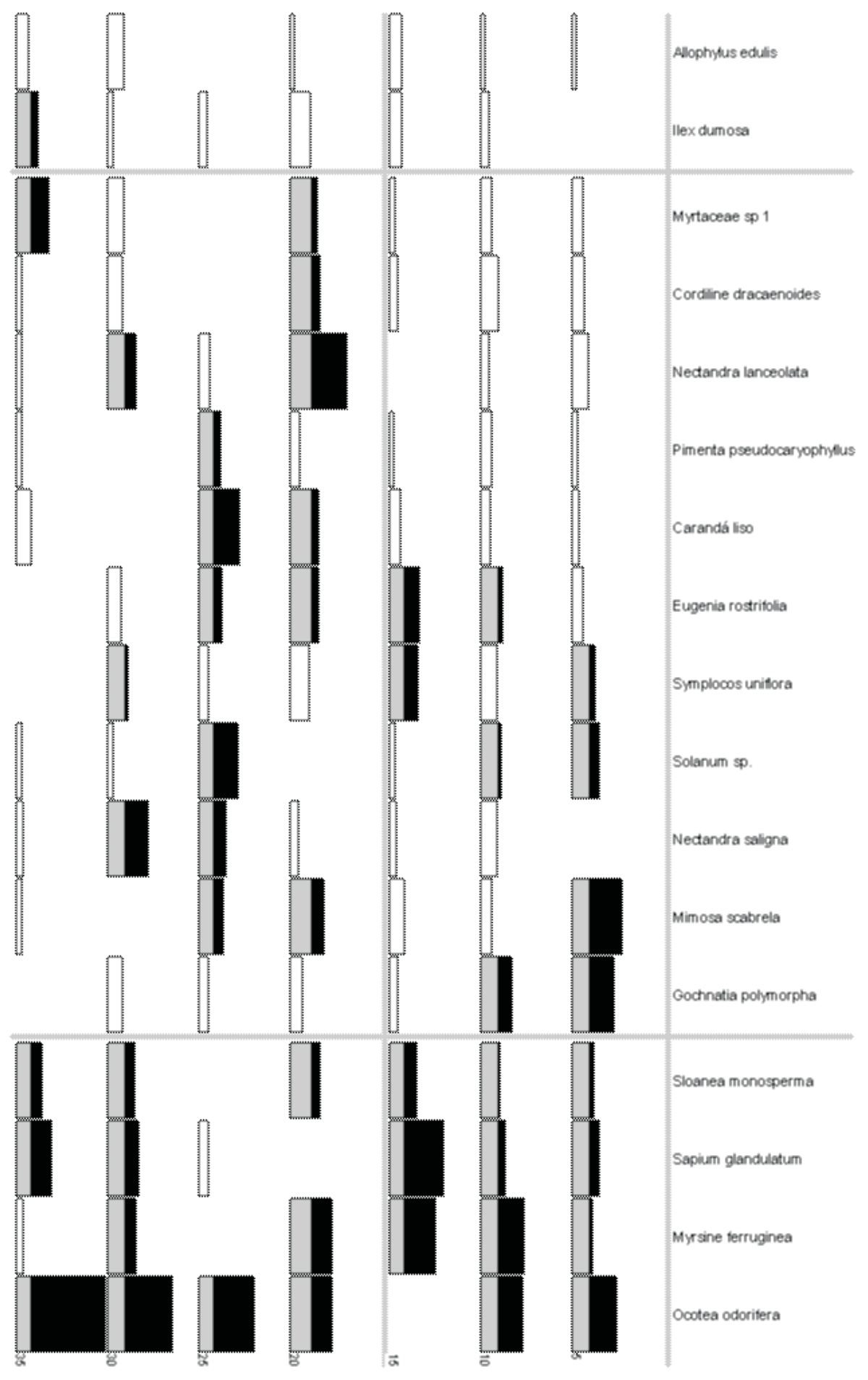




\section{Referências}

DUTRA, R. N.; SPERANDIO, M.; COELHO, J. O Método Ward de Agrupamento de Dados e sua Aplicação em Associação com os Mapas AutoOrganizáveis de Kohonen. In: WORKCOMP SUL WORKSHOP DE CIÊNCIAS DA COMPUTAÇÃO E SISTEMAS DA INFORMAÇÃO DA REGIÃO SUL,1., Florianópolis, 2004. Anais... Florianópolis: UNISUL, 2004 .

IAPAR. Dados agroclimáticos. Disponível em: <Acesso em: 15 out.2005" http://www.pr.gov.br/ iapar>. Acesso em: 15 out.2005.

KOEHLER, A.; PÉLLICO NETTO, S.; SANQUETTA, C. R. Análise da estrutura de uma Floresta Ombrófila Mista semidevastada, Fazenda Gralha Azul, Região Metropolitana de Curitiba, com implicações ao manejo. Revista Acadêmica da PUCPR, Curitiba, n. 1, p. 37-60, 1998.

LAURENCE, W. F., et al. Rainforest fragmentation and the dynamics of Amazonian tree communities. Ecology, Washington, v. 79, n. 6, p. 2032-2040, 1998.
LORENZI, H. Árvores brasileiras: manual de identificação e cultivo de plantas arbóreas do Brasil. 4. ed. Instituto Plantarum, São Paulo, SP: Nova Odessa, v. 1, 2002.

MEFFE, G. K. Principles of conservation biology. Sunderland: Sinauer Associates, 1994.

PRIMACK, R. B., RODRIGUES, E. Biologia da conservação. Londrina: [s. n.], 2001.

RODRIGUES, E. Edge effects on the regeneration of forest fragments in south Brazil. Cambridge, 1998. 172 f. Tese (Doutorado em Biologia) Department of Organismic and Evolutionary Biology, Harvard University, 1998.

TABARELLI, M.; SILVA, J. M. C. GASCON, C. Forest Fragmentation, synergisms and the impoverishment of neotropical forests. Biodiversity and conservation, Netherlands, v. 13, p. 1419-1425, 2004 .

VALENTIN, J. L. Ecologia Numérica: Uma introdução à análise multivariada de dados ecológicos. Rio de Janeiro, RJ: Interciência. 2000. 